Bundesgesundheitsbl 2019 $\cdot 62: 706-712$ https://doi.org/10.1007/s00103-019-02946-6 Online publiziert: 26. April 2019

(c) Der/die Autor(en) 2019

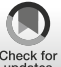

Sebastian Graf von Kielmansegg ${ }^{1}$ Norbert Benda $\cdot$ Guido Grass ${ }^{3}$. Thomas Sudhop ${ }^{2}$

${ }^{1}$ Rechtswissenschaftliche Fakultät, Institut für Öffentliches Wirtschaftsrecht, Christian-AlbrechtsUniversität zu Kiel, Kiel, Deutschland

${ }^{2}$ Bundesinstitut für Arzneimittel und Medizinprodukte (BfArM), Bonn, Deutschland

${ }^{3}$ Ethikkommission der Medizinischen Fakultät, Universität zu Köln, Köln, Deutschland

\title{
Die Rolle von Ethikkommissionen bei der Bewertung klinischer Arzneimittelprüfungen
}

wandelt. Ihre Wurzeln liegen in der kollegialen Beratungsfunktion, die seit 1975 in der Deklaration von Helsinki des Weltärztebundes [1] vorgesehen ist und seit 1985 in das deutsche Berufsrecht übernommen wurde. Charakteristisch für dieses Modell ist die Beschränkung auf eine Beratungspflicht, wie sie bis heute in den ärztlichen Berufsordnungen auf der Basis von $₫ 15$ der Musterberufsordnung verankert ist [2]. Der Tenor des Votums ist in diesem ursprünglichen Modell nicht rechtsverbindlich, sodass eine ablehnende Stellungnahme in rechtlicher Hinsicht nicht zur Unzulässigkeit des Forschungsvorhabens führt. Die Kontrolle klinischer Forschung erscheint hier als Aufgabe der ärztlichen Selbstverwaltung und kollegialen Beratung. Diese Wurzeln im ärztlichen Standesrecht haben bis heute spürbare Nachwirkungen in der Struktur und im Selbstverständnis der Ethikkommissionen.

Für den Bereich der klinischen Forschung mit Arzneimitteln (und Medizinprodukten) hat sich dieses Bild jedoch grundlegend verändert. Die immer feinmaschigere staatliche Regulierung des Arzneimittelrechts beförderte die Ethikkommissionen aus den Gefilden der berufsethischen und -rechtlichen Beratung in die Welt des Ordnungsrechts. Dabei spielten und spielen auch europarechtliche Vorgaben eine maßgebliche Rolle. Schon das AMG in der Fassung von 1994 [3] kombinierte auf eigentümliche und komplizierte Weise ein Anzeigeverfahren bei der Bundesoberbehör- de mit einem vorgeschalteten Zustimmungsverfahren der Ethikkommission. Im Ergebnis war eine klinische Prüfung nach dieser Regelung unzulässig, wenn sie kumulativ von Ethikkommission und Bundesoberbehörde abgelehnt wurde der Tenor des Ethikvotums erhielt damit erstmals eine gewisse rechtliche Relevanz. Die entscheidende Wendung kam aber mit der 12. AMG-Novelle im Jahr 2004, die in Umsetzung der europäischen Richtlinie 2001/20/EG über die Anwendung der guten klinischen Praxis (GCP) das bis heute geltende Modell eingeführt hat. Damit wurde auch die europäische Definition einer klinischen Arzneimittelprüfung in das AMG aufgenommen: Eine klinische Prüfung ist gemäß $₫ 4$ Abs. 23 Satz 1 AMG jede am Menschen durchgeführte Untersuchung, die dazu bestimmt ist, klinische oder pharmakologische Wirkungen, Nebenwirkungen oder die Pharmakokinetik zu untersuchen, mit dem Ziel, sich von der Unbedenklichkeit oder Wirksamkeit der Arzneimittel zu überzeugen. Klinische Prüfungen bedürfen nun einer vorherigen Genehmigung durch die zuständige Bundesoberbehörde wie auch der vorherigen zustimmenden Bewertung durch die zuständige Ethikkommission.

Eine klinische Arzneimittelprüfung darf seither nur noch mit Zustimmung der zuständigen - nach Landesrecht gebildeten - Ethikkommission und einer Genehmigung durch die zuständige Bundesoberbehörde begonnen werden. Der Vorbehalt der kumulativen Ableh- 
nung hat sich in einen kumulativen Zustimmungsvorbehalt umgekehrt [4].

Damit sind Ethikkommissionen bei klinischen Arzneimittelstudien vollständig in die ordnungsrechtliche Regulierung des Arzneimittelrechts integriert. Das Votum der Ethikkommission hat (spätestens) seit der 12. AMG-Novelle die Rechtsnatur eines Verwaltungsaktes im Sinne des $₫ 35$ des Verwaltungsverfahrensgesetzes (VwVfG), weil es die verbindliche Setzung einer Rechtsfolge enthält [5]. Bei Erteilung der zustimmenden Bewertung wird die Durchführung der klinischen Prüfung - vorbehaltlich der ebenfalls erforderlichen Genehmigung durch die Bundesoberbehörde gestattet; bei ihrer Versagung ist die klinische Prüfung rechtlich unzulässig. Dieser Verwaltungsakt hat weitgehend die Funktion und Wirkung einer Genehmigung, wenngleich die im Verwaltungsrecht ungewöhnliche Bezeichnung als "zustimmende Bewertung" einen etwas eingeschränkteren rechtlichen Gehalt andeutet. Konkret: Die zustimmende Bewertung hat eine formelle Gestattungswirkung, sie hebt also die der klinischen Prüfung entgegenstehende präventive gesetzliche Durchführungssperre (partiell) auf. Eine darüber hinausgehende materielle "Legalisierungswirkung“, wie sie für verwaltungsrechtliche Genehmigungen charakteristisch ist, kommt dem Ethikvotum hingegen nicht zu. Das bedeutet, dass das Ethikvotum keine verbindliche Aussage über die materielle Rechtmäßigkeit der klinischen Prüfung trifft, die andere Stellen bei zukünftigen Entscheidungen binden könnte [6].

Indem die Ethikkommissionen nach $\$ 40$ Abs. 1 S. 2 u. $\$ 42$ Abs. 1 AMG rechtsverbindliche Entscheidungen zu treffen haben, agieren sie insoweit als Behörden. Der Gesetzgeber hat sie in diesem Sinne durchaus treffend als „Patientenschutzorganisation mit Behördencharakter"bezeichnet [7]. Es handelt sich bei ihnen in diesem Kontext um Kollegialorgane im Sinn des $₫ 88$ VwVfG. Ihre Wurzeln im ärztlichen Berufsrecht machen sich vor allem noch an ihrer institutionellen Aufhängung bemerkbar. Der Gesetzgeber hat - mit Ausnahme der Länder Berlin, Bremen und Sachsen-Anhalt - darauf verzichtet, staatliche Ethikkommis- sionen als Teil der unmittelbaren Landesverwaltung einzurichten, sondern überträgt die Aufgaben nach $\$ \$ 40 \mathrm{ff}$. AMG nach wie vor den Ethikkommissionen der Landesärztekammern und Universitäten. Gleichwohl handeln die Kommissionen hier nicht im Rahmen der ärztlichen bzw. universitären Selbstverwaltung, sondern des übertragenen staatlichen Wirkungskreises - oder anders formuliert: Sie agieren nicht standes-, sondern ordnungsrechtlich.

\section{Prüfungsmaßstäbe}

Die Kehrseite der Rechtsverbindlichkeit des Ethikvotums bei AMG-Studien ist der sprunghafte Anstieg der gesetzlichen Regelungsdichte in $\$ \$ 40-42 \mathrm{a}$ AMG und der GCP-Verordnung (GCP-V) sowie die gesetzliche Festschreibung der Prüfungs- und Entscheidungsmaßstäbe. Die Liste der möglichen Versagungsgründe wird in $\$ 42$ Abs. 1 S. 7 AMG abschließend festgelegt, vor allem durch Verweis auf die in $\$ \$ 40,41$ AMG aufgeführten formellen und materiellen Voraussetzungen der klinischen Prüfung. Dazu zählen namentlich die ärztliche Vertretbarkeit der Nutzen-Risiko-Relation, das Einwilligungsverfahren, die Qualifikation bzw. Eignung von Prüfern und Prüfzentren, das Vorliegen der Prüferinformation sowie die Probandenversicherung. $\mathrm{Zu}$ den versagungsrelevanten Kriterien gehört außerdem, dass die Prüfungsunterlagen dem Stand der wissenschaftlichen Erkenntnisse entsprechen und geeignet sind, den Nachweis der Unbedenklichkeit oder Wirksamkeit des Arzneimittels zu erbringen. Schließlich bildet - verfahrensrechtlich - auch die Unvollständigkeit der vorgelegten Unterlagen einen Versagungsgrund. Die von der Ethikkommission im Detail zu prüfenden Elemente eines Antrags auf Bewertung einer klinischen Prüfung sind in • Tab. 1 aufgeführt.

Dieser Grundsatz entspricht dem verwaltungsrechtlichen Prinzip des präventiven Verbotes mit Erlaubnisvorbehalt, wonach eine Genehmigung zu erteilen ist, wenn keine gesetzlichen Hinderungsgründe vorliegen. Daraus folgt zunächst einmal, dass die Entscheidung über die Erteilung oder Versagung der zustim- menden Bewertung nicht im Ermessen der Kommission steht, sondern eine gebundene ist. Zum Zweiten ergibt sich daraus, dass die Entscheidung nicht auf rechtliche und ethische Überlegungen jenseits des gesetzlichen Versagungskataloges gestützt werden kann. Das schließt es zwar nicht aus, den Antragstellern unverbindliche Hinweise oder Empfehlungen zu geben. Der rechtsverbindliche Tenor der Entscheidung jedoch - Zustimmung oder Versagung - muss sich ausschließlich danach richten, ob einer der gesetzlichen Versagungsgründe vorliegt oder nicht.

In der Praxis der Ethikkommissionen bedeutet das, dass im Vergleich zur herkömmlichen berufsrechtlichen Beratung die formaljuristischen Fragen weit in den Vordergrund gerückt sind, während die namensgebende ethische Perspektive nur noch im gesetzlichen Rahmen zum Zuge kommen kann. Das ist eine Entwicklung, die in den Ethikkommissionen mit beträchtlichem Unbehagen gesehen wird. Sie beruht allerdings weniger auf der Rechtsverbindlichkeit des Votums und dem abschließenden Katalog der Versagungsgründe als auf der Unzahl formaler Vorgaben, die bei der Bewertung abgearbeitet werden muss.

In Wirklichkeit ist dieser Gegensatz jedoch nicht so scharf, wie er oftmals wahrgenommen wird, denn $\mathrm{zu}$ den rechtlichen Bewertungsmaßstäben und Versagungsgründen gehört auch die (ggf. fehlende) ärztliche Vertretbarkeit der Nutzen-Risiko-Relation, wie sie in $\$ 40$ Abs. 1 S. 3 Nr. 2 AMG verlangt ist. Dabei handelt es sich um einen gesetzlichen Generalverweis auf einen Abwägungsvorgang, der durch ethische Maßstäbe und ärztliche Erfahrung geprägt ist und der seit jeher das Herzstück der ethischen Beurteilung durch Ethikkommissionen bildet. Hier bilden Recht und Ethik also keinen Gegensatz, sondern das Recht inkorporiert weitgehend ungefiltert einen ethischen Diskurs und Beurteilungsmaßstab. Zudem ist den Ethikkommissionen nach den allgemeinen Regeln verwaltungsrechtlicher Dogmatik in diesem Punkt ein weitgehender Beurteilungsspielraum zuzugestehen, der einer etwaigen gerichtlichen Kontrolle enge Grenzen setzt. 
Bundesgesundheitsbl 2019·62:706-712 https://doi.org/10.1007/s00103-019-02946-6

(c) Der/die Autor(en) 2019

\section{S. Graf von Kielmansegg · N. Benda · G. Grass · T. Sudhop}

\section{Die Rolle von Ethikkommissionen bei der Bewertung klinischer Arzneimittelprüfungen}

\section{Zusammenfassung}

Die Rolle von Ethikkommissionen bei der Bewertung klinischer Arzneimittelprüfungen hat sich im Laufe der Jahre gewandelt. Aus den kollegialen Beratungsgremien wurden Patientenschutzorganisationen mit Behördencharakter. Während das ärztliche Berufsrecht in Deutschland bei biomedizinischer Forschung am Menschen lediglich eine Beratungspflicht für Ärzte vorsieht, bei der eine ablehnende Stellungnahme in rechtlicher Hinsicht nicht zu einer Unzulässigkeit des Forschungsvorhabens führt, verlangt das Arzneimittelgesetz (AMG) eine zustimmende Bewertung durch die zuständige Ethikkommission.

Das AMG definiert sowohl die durch die Ethikkommission zu prüfenden Elemente eines Antrages für eine klinische Arzneimittelprüfung als auch abschließend die Gründe, anhand derer die zustimmende Bewertung versagt werden kann. Ethikkommissionen, die Anträge gemäß AMG bewerten, müssen nach Landesrecht gebildet sein und sind interdisziplinär mit Fachärzten, Juristen und Methodikern besetzt. Wesentliche Bewertungsinhalte sind ein ärztlich vertretbares Nutzen-Risiko-Verhältnis, die Angemessenheit der verwendeten Methoden einschließlich biometrischer Aspekte, die Anforderungen an die Studienteilnehmer, wie z. B. Einwilligungsfähigkeit, die Eignung der Prüfer und Prüfeinrichtungen sowie die Angemessenheit der schriftlichen Informationen, mit denen
Studienteilnehmer informiert werden und sie ihre Einwilligung erteilen sollen.

Trotz der bereits jetzt hohen Regelungsdichte werden zukünftig mit der Anwendbarkeit der Verordnung (EU) Nr. 536/2014 die Anforderungen an Zusammensetzung und Arbeitsweise von Ethikkommissionen noch detaillierter gesetzlich festgelegt mit dem Ziel, die Bewertung klinischer Arzneimittelprüfungen in der EU weiter zu harmonisieren.

\section{Schlüsselwörter}

Klinische Studien · Klinische Arzneimittelprüfungen · Arzneimittelgesetz (AMG) · Ethikkommissionen

\section{Ethics committees in clinical trials involving medicinal products}

\section{Abstract}

Over the years, the role of ethics committees (ECs) in the review process of clinical trial applications (CTAs) has changed from being a collegial advisory body to a patient protection organisation with an authority character. While the law governing the medical profession in Germany only provides for an obligation for physicians to ask for an EC review in biomedical research on human beings, a negative opinion on the CTA does not lead to the inadmissibility of the research project from a legal point of view. In contrast, the German Medicinal Product Act (Arzneimittelgesetz, AMG) requires a favourable opinion as an approving assessment by the competent $\mathrm{EC}$.
The AMG defines both the elements of a clinical trial application to be reviewed by the EC as well as the principle grounds of nonacceptance to reject a favourable opinion. ECs that assess CTAs must be constituted in accordance with the state law and must be composed of interdisciplinary medical specialists, lawyers and methodologists. The main assessment criteria are a medically acceptable risk-benefit ratio, the appropriateness of the methods used, including biometric aspects, the requirements to be met by the study participants, such as their ability to give consent, the suitability of the investigators and trial facilities as well as the appropriateness of the written information with which the study participants are to be informed and give their consent. In spite of the already high degree of regulation, the applicability of the European Clinical Trial Regulation will result in even more detailed legal requirements for the composition and working procedures of an $\mathrm{EC}$ with the aim of further harmonising the assessment of CTAs in the EU.

Keywords

Clinical studies - Clinical trials with medicina products - German Medicinal Products Act (AMG) · Ethics committees
$\mathrm{Zu}$ den von der Ethikkommission zu prüfenden Zulässigkeitsvoraussetzungen einer klinischen Prüfung zählt auch die datenschutzrechtliche Aufklärung und Einwilligung nach $\$ 40$ Abs. 2a AMG. Zunehmend in die Diskussion gerät demgegenüber, inwieweit auch die sonstigen Anforderungen des allgemeinen Datenschutzrechts für das Ethikvotum zu berücksichtigen sind - insbesondere seit Wirksamwerden der Datenschutzgrundverordnung im Mai 2018. Da der gesetzliche Katalog der Versagungsgründe das allgemeine Datenschutzrecht nicht einschließt, ist das nach jetziger Rechtslage richtigerweise $\mathrm{zu}$ verneinen, zumindest soweit nicht die datenschutzrechtliche Information und Einwilligung selbst betroffen sind. Die Grenzziehung bleibt im Einzelfall freilich schwierig und die Neuregelung des Regimes klinischer Prüfungen durch die Verordnung (EU) Nr. 536/2014 wird auch in diesem Punkt die rechtlichen Rahmenbedingungen zukünftig verschieben [8].

\section{Zusammensetzung einer Ethikkommission}

Ethikkommissionen, die klinische Prüfungen gemäß AMG bewerten, müssen nach den Vorgaben in $\$ 42$ Abs. 1 AMG nach Landesrecht gebildet sein. Die Voten privatrechtlicher Ethikkommissionen können nicht als zustimmende Bewertung im Sinne des $\$ 40$ Abs. 1 S. 2 AMG gewertet werden. Die Zusammensetzung, Bildung und Finanzierung von Ethikkommissionen sind derzeit noch ausschließlich in den jeweiligen Rege- 
Tab. 1 Von der Ethikkommission zu prüfende Elemente bei der Bewertung einer klinischen Arzneimittelprüfung gemäß Arzneimittelgesetz (AMG)

\section{Element}

Bezug im AMG

Vollständigkeit der vorgelegten Unterlagen

$\S 42$ Abs. 1 S. 7 Nr. 1

Angemessenheit der Methodik gemessen am Stand der wissenschaftlichen Erkenntnisse einschließlich der Eignung der klinischen Prüfung zum Nachweis der Unbedenklichkeit oder Wirksamkeit der Prüfarzneimittel ggf. einschließlich genderrelevanter Unterschiede

Nutzen-Risiko-Bewertung unter Abwägung individueller Risiken und der Bedeutung des Arzneimittels für die Humanmedizin

$\S 42$ Abs. 1 S. 7 Nr. 3 i.V.m. $\S 40$ Abs. 1 S. 3. Nr. 2

Vertretbarkeit möglicher Risiken durch Arzneimittel, die gentechnisch veränderte Organismen enthalten, auf die Gesundheit Dritter und die Umwelt

Anforderung an die Studienteilnehmer:Volljährigkeit, Geschäftsfähigkeit, keine gerichtlich oder behördlich angeordnete Unterbringung in einer Anstalt, Aufklärung der Prüfungsteilnehmer gemäß $\S 40$ Abs. 2, schriftliche Einwilligung zur Teilnahme und zur Datenerhebung und -verarbeitung von Gesundheitsdaten

Eignung der Prüfeinrichtung und der Prüfer einschließlich Angemessenheit der erforderlichen Prüfererfahrung

Angemessenheit der pharmakologisch-toxikologischen Prüfung der Prüfarzneimittel und Information der Prüfer darüber, in der Regel durch Überprüfung der Prüferinformation (Investigator's Brochure)

Notwendigkeit und Angemessenheit der Versicherung für Prüfungsteilnehmer

Medizinische/zahnmedizinische Versorgung durch einen Arzt bzw. Zahnarzt

$\S 42$ Abs. 1 S. 7 Nr. 3 i.V.m. $\S 40$ Abs. 1 S. 3. Nr. $2 a$

$\S 42$ Abs. 1 S. 7 Nr. 3 i.V.m. $\S 40$ Abs. 1 S. 3. Nr. 3 und 4

Prüfung der allgemeinen Vorschriften für minderjährige Prüfungsteilnehmer einschließlich Aufklärung und Einwilligung

Prüfung der besonderen Vorgaben für Prüfungsteilnehmer, die an der Krankheit leiden, für die das Prüfpräparat intendiert ist, einschließlich der Regelungen für Notfallsituationen, Minderjährige und nichteinwilligungsfähige Erwachsene

lungen der Bundesländer, z.B. in den Heilberufegesetzen der Länder, oder in den Satzungen der Ethikkommissionen verankert. Das AMG fordert explizit lediglich die Hinzuziehung von Sachverständigen oder Gutachten bei klinischen Prüfungen, die Minderjährige beinhalten, sofern die Kommission über keine eigenen Experten auf diesem Gebiet verfügt, und bei klinischen Prüfungen von xenogenen Arzneimitteln oder Gentherapeutika. Jede Ethikkommission, die das Bewertungsverfahren gemäß AMG vornimmt, verfügt über Fachärzte sowie juristische Experten. Meist sind zusätzlich auch medizinische Laien und in der medizinischen Ethik erfahrene Personen, teilweise auch Seelsorger, Mitglieder von Ethikkommissionen. Während Biometriker oder Methodiker früher eher selten in Kommissionen vertreten waren, verfügen derzeit immer mehr Kommissionen
Facharzt für klinische Pharmakologie oder für Pharmakologie und Toxikologie), sowie eines Laien. Alle derzeit beim BfArM registrierten Ethikkommissionen erfüllen diese Voraussetzung bereits jetzt [9].

\section{Arbeitsweise einer Ethikkommission}

Die Arbeit von Ethikkommissionen erfolgt meist in Form von Kommissionssitzungen, an denen die einzelnen Anträge auf Bewertung einer klinischen Prüfung oder entsprechende Änderungsanzeigen diskutiert und anschließend votiert werden. Kommissionssitzungen werden in der Regel von einem Vorsitz geleitet, der nach der jeweiligen Geschäftsordnung bestimmt wird. Der Vorsitz fasst in der Regel die Diskussion zusammen, formuliert in einem Beschlussvorschlag das Votum oder Nachforderungen sprachlich vor und stellt diesen in der Kommission zur Abstimmung. Sofern ein formal vollständiger und ordnungsgemäßer Antrag eingereicht wurde, muss die Kommission den Antrag bei klinischen Arzneimittelprüfungen innerhalb von höchstens 60 Tagen bewerten. Während dieser Zeit kann sie nur ein einziges Mal zusätzliche Informationen vom Sponsor anfordern. Tut sie dies, wird die Frist bis zum Eingang der zusätzlichen Informationen gehemmt. Bei rein monozentrischen klinischen Prüfungen wird die Bewertungsfrist auf 30 Tage verkürzt. Bei einer klinischen Prüfung der Phase I, die als Teil eines Entwicklungsprogramms auf einer von dieser Ethikkommission bereits zustimmend bewerteten klinischen Prüfung basiert, verkürzt sich die Frist auf 14 Tage. Für die Bewertung xenogener Arzneimittel sieht das AMG keine Frist vor. Spätestens am Ende der jeweiligen Frist übermittelt die Ethikkommission dem Sponsor und der zuständigen Bundesoberbehörde ihre mit Gründen versehene Bewertung. Aufgrund der kurzen Fristen hat sich in den meisten Kommissionen ein 14-tägiger, teilweise gar ein 7-tägiger Sitzungsturnus etabliert. 


\section{Zuständigkeit einer Ethikkommission}

Die Zuständigkeit einer Ethikkommission ergibt sich nach $\$ 42$ Abs. 1 AMG anhand des Sitzes des Prüfers bzw. bei mehreren Prüfzentren in Deutschland durch den Sitz des Leiters der klinischen Prüfung. Die für den Prüfer bzw. den Leiter der klinischen Prüfung nach Landesrecht zuständige Ethikkommission ist für die Administration des Verfahrens und die (zustimmende) Bewertung des Antrages zuständig. Das heißt, sie bewertet den Antrag an und für sich und die Eignung der Prüfer und Prüfzentren für den jeweiligen Antrag. Bei multizentrischen Prüfungen mit Prüfern und Zentren außerhalb des Zuständigkeitsbereichs der zuständigen Ethikkommissionen bezieht sie sich bei der Bewertung der Prüfer- und Zentrumseignung auf die Stellungnahme der jeweils für diese Zentren zuständigen Ethikkommissionen, die am Verfahren als sogenannte beteiligte Ethikkommissionen involviert sind. Bei diesen multizentrischen klinischen Prüfungen bewertet die zuständige Ethikkommission federführend den Antrag im Benehmen mit den beteiligten Ethikkommissionen. Dieses Benehmensverfahren verlangt insbesondere in Bezug auf die grundsätzliche Bewertung des Antrages nur eine Beteiligung im Benehmen, aber kein Einvernehmen zwischen zuständiger federführender und beteiligter Ethikkommission. Die Gesamtentscheidung obliegt der zuständigen federführenden Ethikkommission. Dieses Verfahren der Zuordnung wird zukünftig mit Anwendbarkeit der Verordnung (EU) Nr. 526/2014 - voraussichtlich nicht vor dem Jahr 2020 durch einen jährlichen Geschäftsverteilungsplan abgelöst werden, bei dem die zuständige Kommission gleichsam zufällig zugeordnet wird [8].

\section{Medizinische Bewertung}

Die medizinische Bewertung eines Antrags erfolgt im Wesentlichen auf $\mathrm{Ba}$ sis der Angaben im Prüfplan und der Prüferinformation. Letztere enthält u.a. die Ergebnisse der pharmakologisch-toxikologischen Prüfung des Prüfpräparates sowie eine Zusammenfassung der be- reits am Menschen durchgeführten klinischen Prüfungen und eine allgemeine Nutzen-Risiko-Bewertung des Prüfpräparates an sich, unabhängig von der einzelnen klinischen Prüfung. Neben der Bewertung der Studienrationale sind besonders die Überprüfung der Geeignetheit der Studienpopulation anhand der im Prüfplan angegebenen Ein- und Ausschlusskriterien sowie die Angemessenheit der Vergleichsbehandlung Gegenstand der medizinischen Bewertung. Dabei steht im Wesentlichen die Nutzen-Risiko-Bewertung der klinischen Prüfung im Vordergrund, die sich in $\$ 40$ Abs. 1 S. 3 Nr. 2 AMG an der ärztlichen Vertretbarkeit der vorhersehbaren Risiken und Nachteile gegenüber dem Nutzen für die Prüfungsteilnehmer und an der voraussichtlichen Bedeutung des Arzneimittels für die Heilkunde orientiert. Als Grundlage für die medizin-ethische Bewertung, z. B. bei Gebrauch von Placebos, dient den Ethikkommissionen die Deklaration von Helsinki des Weltärztebundes. Auch wenn dies nicht unmittelbar im AMG gefordert ist, werden diese Grundprinzipien durch den Bezug in $\$ 40$ Abs. 1 AMG auf die gute klinische Praxis nach Maßgabe der Richtlinie 2001/20/EG [10] angesprochen, welche selbst in Erwägungsgrund 2 sowie in Artikel 3 der Richtlinie 2005/28/EG unmittelbar Bezug auf die Deklaration von Helsinki nehmen. Neben der Prüfung des Equipoise-Prinzips - also der grundsätzlichen A-priori-Annahme der Gleichwertigkeit der Intervention aller Studienarme als ethische Voraussetzung zur Randomisierung bei kontrollierten randomisierten Studien [11-13] - werden insbesondere auch der individuelle Belastungsgrad sowie die möglichen individuellen Risiken für die Studienteilnehmer von den Ethikkommissionen unter medizinischen $\mathrm{Ge}$ sichtspunkten geprüft. Insbesondere für Minderjährige und nichteinwilligungsfähige Erwachsene hat der Gesetzgeber strikte Vorschriften erlassen, die auf eine Minimierung der Risiken und Belastungen abzielen. Eine besondere Prüfung hat der Gesetzgeber den Ethikkommissionen bei der Bewertung klinischer Prüfung mit Minderjährigen auferlegt. Hier$\mathrm{zu}$ muss die Kommission mit eigener oder - sofern nicht direkt vorhanden - mit hinzugezogener pädiatrischer Expertise speziell auch die ethischen und psychosozialen Besonderheiten dieser Population bei der Bewertung berücksichtigen, wie sie z. B. im entsprechenden Guidance-Dokument der EU-Kommission diskutiert werden [14]. Umfasst die klinische Prüfung zusätzlich auch die Anwendung von ionisierenden Strahlen, z. B. im Rahmen der Diagnostik, aber auch der Therapie, fällt der Ethikkommission zusätzlich noch die strahlenschutzrechtliche Bewertung $\mathrm{zu}$, sofern sie sich als entsprechende Kommission beim Bundesamt für Strahlenschutz hat registrieren lassen [15].

\section{Methodische und biometrische Bewertung}

Gemäß $\$ 42$ Abs. 1 S. 7 Nr. 2 AMG kann die Genehmigung einer klinischen Prüfung versagt werden, falls „die vorgelegten Unterlagen ... nicht dem Stand der wissenschaftlichen Erkenntnisse entsprechen, insbesondere die klinische Prüfung ungeeignet ist, den Nachweis der Unbedenklichkeit oder Wirksamkeit eines Arzneimittels ... zu erbringen“.

Eine klinische Prüfung kann unterschiedliche Ziele und unterschiedliche wissenschaftliche Fragen verfolgen. Das geplante Studiendesign einschließlich der statistischen Auswertung muss in der Lage sein, diese Fragen hinreichend und entsprechend dem Stand der wissenschaftlichen Erkenntnisse zu beantworten. Da davon auszugehen ist, dass eine klinische Prüfung in der Regel ein gewisses Risiko für die Studienteilnehmer darstellt, sollte der zu erwartende Informationsgewinn, der langfristig zukünftigen Patienten zugutekommen soll, relevant genug sein, um dieses Risiko zu rechtfertigen.

Die statistische Analyse zusammen mit dem Studiendesign der klinischen Studie kann im methodischen Sinn als ein Entscheidungsproblem aufgefasst werden, das ausgehend von einer wissenschaftlichen Fragestellung eine Regel bereitstellt, welche spezifiziert, wie Schlussfolgerungen für eine größere Population von den zu gewinnenden Daten abgeleitet werden (statistische Tests oder Schätzverfahren). Zum Beispiel wird 
in einer konfirmatorischen klinischen Prüfung die Wirksamkeit eines neuen Medikaments im Vergleich zu Placebo gefolgert, falls der entsprechende statistische Test eine Signifikanz liefert. Andere Studien erlauben als Schlussfolgerung zumindest die Schätzung wichtiger Parameter, z.B. die einer Dosis-WirkungsBeziehung, und die Generierung von Hypothesen, welche in weiteren Studien untersucht werden können. Diese Studien werden in der Regel als explorativ bezeichnet.

Ziel der biometrischen Bewertung einer AMG-Studie muss es sein, zu bewerten, ob das statistische Entscheidungsverfahren dem Stand der wissenschaftlichen Erkenntnisse entspricht und es erlaubt, sinnvolle Schlüsse bezüglich der Unbedenklichkeit oder Wirksamkeit eines Arzneimittels zu ziehen. Solche Entscheidungsregeln, insbesondere statistische Tests, sind für ein gegebenes Problem nicht eindeutig und müssen deswegen im Prüfplan präspezifiziert werden. Ebenso liefern solche Regeln auch falsche Entscheidungen (z. B. der statistische Fehler, sich mit einem signifikanten Testergebnis für die Überlegenheit eines Arzneimittels gegenüber Placebo zu entscheiden, obwohl diese in Wirklichkeit nicht gegeben ist). Die Bewertung muss beurteilen, ob die Eigenschaften der Entscheidungsregel akzeptabel sind und ob die vorhandenen Ressourcen effizient genutzt werden. Eine konfirmatorische $\mathrm{Zu}$ lassungsstudie zum Nachweis der Wirksamkeit eines neuen Medikaments verlangt z. B., dass die Rate der falschen Entscheidungen zugunsten der Wirksamkeit durch 2,5\% (d.h. der einseitige Fehler erster Art) begrenzt sein muss.

Wann sind nun biometrische Mängel versagungsrelevant? Ausgehend von der wissenschaftlichen Frage der Studie sind hierbei grundsätzlich folgende zwei Fragen von besonderer Relevanz:

1. Entspricht das statistische Verfahren der wissenschaftlichen Fragestellung und weist es die behaupteten oder verlangten Kriterien auf, mit anderen Worten, macht das Entscheidungsverfahren das, was es vorgibt?

2. Ist das Verfahren effizient und aussagekräftig genug, um die klinische Prüfung zu rechtfertigen?
Bestimmte Studien verlangen bestimmte Kriterien, z.B. verlangt eine konfirmatorische Phase-III-Studie in der Regel die Kontrolle der Rate der falsch-positiven Entscheidungen der primären Analyse. Insbesondere können dabei fehlende Präspezifizierung, ungenügende Adjustierung für Mehrfachtestung, offensichtlich falsche Modellannahmen oder mangelhafte Behandlung fehlender Daten dazu führen, dass diese Rate nicht eingehalten wird, was dazu führt, dass die Studie ihren vorgegebenen Zweck nicht mehr erfüllen kann. Andere Studien, u.a. Studien der frühen klinischen Entwicklung, verlangen nicht notwendigerweise dasselbe Kriterium. Zwangsläufig schließt die Beurteilung des statistischen Entscheidungsverfahrens das Studiendesign mit ein, da die Eigenschaften des Verfahrens von der Stichprobenziehung, also dem Design, abhängen.

Eine falsche Fallzahlberechnung oder ein ineffizientes Test- oder Schätzverfahren beeinflussen nicht notwendigerweise die Validität des Verfahrens, können aber dazu führen, dass die Studie nur mit geringer Wahrscheinlichkeit bzw. mit unzureichender Präzision die vorgegebene Fragestellung beantworten kann, was als zu behebender Mangel benannt werden muss. Effizienz hängt allerdings meist von unterschiedlichen Annahmen und Zielen ab, sodass bestimmte Verfahren nicht per se abgelehnt werden können.

Insgesamt beurteilt die biometrische Bewertung das Studiendesign und die statistische Analyse als datengestütztes Entscheidungsverfahren hinsichtlich ihrer Fähigkeit, die angegebene wissenschaftliche Frage adäquat zu beantworten, und ob die erforderlichen Eigenschaften des Verfahrens gegeben sind. Hierbei ist das Entscheidungsverfahren nicht eindeutig, sollte aber durch sinnvolle Annahmen begründet werden können. Manche statistischen Mängel der vorgelegten klinischen Prüfung werden durch einfache Modifikationen zu beheben sein (z.B. Anwendung eines anderen statistischen Tests oder einer adäquaten Berücksichtigung fehlender Werte), andere werden ein anderes Studiendesign erfordern (z.B. randomisierte Studie statt einer einarmigen Studie).
Zulassungsstudien der Phase III, bei denen viele Mängel notwendigerweise zur Ablehnung der Studie führen, werden sicherlich hinsichtlich der strikteren Kriterien einer konfirmatorischen Studie strenger bewertet als eher explorative Studien einer frühen Phase oder Studien außerhalb eines klinischen Entwicklungsprogramms. Dennoch muss in allen Fällen das potenzielle Risiko einer klinischen Prüfung durch den zu erwartenden Erkenntnisgewinn durch das statistische Verfahren gerechtfertigt sein. Als Beispiel einer nichtkonfirmatorischen Studie seien risikobehaftete First-in-man-Dosiseskalationsstudien genannt, in denen die Effizienz und der eigentliche Nutzen erheblich von dem vorgeschlagenen Design abhängen können.

\section{Fazit}

Die Aufgaben einer Ethikkommission bei der Bewertung eines Antrags auf klinische Prüfung eines Arzneimittels sind vielfältig und durch zahlreiche Vorschriften im AMG und der nachfolgenden GCP-Verordnung rechtlich geregelt. Insbesondere die Ablehnung einer klinischen Arzneimittelprüfung muss sich auf einen der gesetzlich festgelegten Versagungsgründe beziehen. Andere als die im AMG aufgeführten Gründe dürfen nicht herangezogen werden. Als interdisziplinär besetztes unabhängiges Expertengremium übernehmen Ethikkommissionen bei der Bewertung von klinischen Arzneimittelprüfungen eine Behördenfunktion und sind daher nach dem Recht des Landes, in dem sie ihren Sitz haben, errichtet und arbeiten nach entsprechenden heilberufsrechtlichen Vorgaben in den einzelnen Bundesländern. Neben der Prüfung der Eignung der Prüfer und Prüfeinrichtungen, bei der im Falle multizentrischer klinischer Prüfungen ggf. auch weitere Ethikkommissionen zu beteiligen sind, prüfen die Ethikkommissionen im Wesentlichen den Prüfplan, die Prüferinformation und das an die Prüfungsteilnehmer ausgegebene Informationsmaterial einschließlich der Einwilligungserklärungen zur Teilnahme und zum Datenschutz. Das Verfahren 
bei den Ethikkommissionen wird sich durch die Anwendung der Verordnung (EU) 536/2014 voraussichtlich im Jahr 2020 deutlich ändern und eine engere Zusammenarbeit zwischen zuständiger Ethikkommission und zuständiger Bundesoberbehörde erfordern.

\section{Korrespondenzadresse}

\section{Thomas Sudhop}

Bundesinstitut für Arzneimittel und

Medizinprodukte (BfArM)

Bonn, Deutschland

thomas.sudhop@bfarm.de

\section{Einhaltung ethischer Richtlinien}

Interessenkonflikt. G. Grass ist Leiter der Geschäftsstelle der Ethikkommission der Universität zu Köln. S. Graf von Kielmansegg ist Mitglied der Ethikkommissionen der Christian-Albrechts-Universität zu Kiel. Beide sind Mitglieder im Vorstand des Arbeitskreises Medizinischer Ethik-Kommissionen in der Bundesrepublik Deutschland e. V.N. Benda und T. Sudhop geben an, dass kein Interessenkonflikt besteht.

Für diesen Beitrag wurden von den Autoren keine Studien an Menschen oder Tieren durchgeführt. Für die aufgeführten Studien gelten die jeweils dort angegebenen ethischen Richtlinien.

Open Access. Dieser Artikel wird unter der Creative Commons Namensnennung 4.0 International Lizenz (http://creativecommons.org/licenses/by/4.0/deed. de) veröffentlicht, welche die Nutzung, Vervielfältigung, Bearbeitung, Verbreitung und Wiedergabe in jeglichem Medium und Format erlaubt, sofern Sie den/die ursprünglichen Autor(en) und die Quelle ordnungsgemäß nennen, einen Linkzur Creative Commons Lizenz beifügen und angeben, ob Änderungen vorgenommen wurden.

\section{Literatur}

1. World Medical Association (2013) Declaration of Helsinki: ethical principles for medical research involving human subjects. JAMA 310:2191-2194

2. Bundesärztekammer (2018) (Muster-)Berufsordnung für die in Deutschland tätigen Ärztinnen und Ärzte. http://www.bundesaerztekammer.de/ fileadmin/user_upload/downloads/pdf-Ordner/ MBO/MBO-AE.pdf. Zugegriffen: 30. November 2018

3. Fünftes Gesetz zur Änderung des Arzneimittelgesetzes vom 9. August 1994. BGBI. I., S. 2071.

4. Zwölftes Gesetzzur Änderung des Arzneimittelgesetzes vom 30. Juli 2004. BGBI.I, S. 2031.

5. Rehmann W (2016) In: Kügel JW, Müller R-G, Hofmann H-P (Hrsg) Arzneimittelgesetz: AMG. Beck, München

6. Kielmansegg S (2008) Die rechtliche Bedeutung der Voten von Ethik-Kommissionen. In: Just, Kindt, Koch (Hrsg) Rechtliche Grundlagen der Beratung von Forschungsvorhaben Kolloquium der Ethik-
Kommission der Albert-Ludwigs-Universität Freiburg, 24.10.2008, S43-62

7. (2003) Gesetzentwurf der Bundesregierung: Entwurfeines Zwölften Gesetzes zur Änderung des Arzneimittelgesetzes. In: BT-Drucksache 15/2109, S. 1-44.

8. Nickel L, Seibel Y, Frech M, Sudhop T (2017) Änderungen des Arzneimittelgesetzes durch die EU-Verordnung zu klinischen Prüfungen. Bundesgesundheitsbl Gesundheitsforschgesundheitsschutz 60:804-811

9. (2018) Bekanntmachung der Liste der gemäß $\S 41 a$ AMG registrierten Ethik-Kommissionen. In: Bundesinstitut für Arzneimittel und Medizinprodukte. https://www.bfarm.de/SharedDocs/ Downloads/DE/Arzneimittel/Zulassung/klinpr/ethikKomm/Liste\%20der\%20registrierten \%20Ethik-Kommissionen.html. Zugegriffen: 30. November 2018

10. (2001) Directive 2001/20/EC of the European parliament and of the council of 4 April 2001 on the approximation of the laws, regulations and administrative provisions of the Member States relating to the implementation of good clinical practice in the conduct of clinical trials on medicinal products for human use. Official Journal of the European Communities, p 34. https://ec.europa. eu/health/sites/health/files/files/eudralex/vol-1/ dir_2001_20/dir_2001_20_en.pdf. Zugegriffen: 30. November 2018

11. Freedman $B$ (1987) Equipoise and the ethics of clinical research. N Engl J Med 317:141-145

12. Miller FG (2006) Equipoise and the ethics of clinical research revisited. Am J Bioeth 6:59-61

13. Miller FG, Brody H (2007) Clinical equipoise and the incoherence of research ethics. J Med Philos 32:151-165

14. European Commission (2008) Ethical considerations for clinical trials on medicinal products conducted with the paediatric population. http://ec.europa.eu/health/sites/health/files/ files/eudralex/vol-10/ethical_considerations_en. pdf 2018/12/06.Zugegriffen:30. November 2018

15. Bundesamt für Strahlenschutz (BfS) (2018) Registrierte Ethikkommissionen. http:// www.bfs.de/DE/themen/ion/anwendungmedizin/forschung/ethikkommissionen/ ethikkommissionen_node.html. Zugegriffen: 30. November 2018 